\title{
AVALIAÇÃO DE DIFERENTES ESPAÇAMENTOS PARA PLANTIO DE MOGNO AFRICANO (Khaya sp.)
}

Marco Antônio Marcelino Bahia'; Sybelle Barreira ${ }^{2}$; Hallefy Souza3; Thiago Augusto Sampaio Teles ${ }^{4}$

${ }^{1}$ Acadêmico em Engenharia Florestal, Grupo de Pesquisa Cerrado, Escola de Agronomia, Universidade Federal de Goiás, Goiânia, Goiás, Brasil. eng.marcobahia@gmail.com.

2 Professora Associada, Setor de Engenharia Florestal, Escola de Agronomia, Universidade Federal de Goiás, Brasil.

${ }^{3}$ Engenheiro Florestal, Mestrando em Ciências Florestais- PPGCF, Faculdade de

Tecnologia, Universidade de Brasília.

${ }^{4}$ Biólogo, Mestre em Agronegócio, Setor de Engenharia Florestal, Escola de Agronomia, Universidade Federal de Goiás, Brasil.

Recebido em: 04/10/2019 - Aprovado em: 30/11/2019 - Publicado em: 15/12/2019 DOl: 10.18677/EnciBio 2019B7

\begin{abstract}
RESUMO
O estudo do desenvolvimento de um plantio florestal sob diferentes espaçamentos é de suma importância tanto para colaborar com a ciência, quanto para maximizar o rendimento do plantio. Esse trabalho teve por objetivo quantificar o desenvolvimento dendrométrico e volumétrico, de aproximadamente 400 árvores divididas em cinco espaçamentos distintos, de dimensões: $6 \times 7 \mathrm{~m}, 6 \times 6 \mathrm{~m}, 5 \times 5 \mathrm{~m}, 4 \times 4 \mathrm{~m}$ e $3 \times 3 \mathrm{~m}$; além de fazer uma análise fitossanitária e de tortuosidade nas árvores de um experimento de aproximadamente 48 meses, instalado no município de Bonfinópolis-GO. O trabalho foi realizado entre os meses de março de 2018 e março de 2019. Em termos gerais - espaçamento $5 \times 5 \mathrm{~m}$ apresentou as maiores médias de DAP, altura e volume individual. Embora não houvesse diferenças significativas entre as médias de ICA para a variável diâmetro, foi possível identificar para as médias de ICA da variável altura, que todos os espaçamentos apresentaram resultados superiores ao apresentado pelo espaçamento $6 \times 6 \mathrm{~m}$. O espaçamento $3 \times 3 \mathrm{~m}$ apresentou maior volume por hectare, em vista da alta densidade populacional.
\end{abstract}

PALAVRAS-CHAVE: Plantios Comerciais; Plantio florestal; Silvicultura.

\section{EVALUATION OF DIFFERENT SPACINGS FOR PLANTING OF AFRICAN MAHOGANY (Khaya sp.) ABSTRACT}

The study of the development of a forest plantation under different spacings is of paramount importance to collaborate with science, and to maximize the yield of planting. This study aimed to quantify the dendrometric and volumetric development of approximately 400 trees divided into 5 distinct spacings, of dimensions: $6 \times 7 \mathrm{~m}$, $6 \times 6 \mathrm{~m}, 5 \times 5 \mathrm{~m}, 4 \times 4 \mathrm{~m}$ and $3 \times 3 \mathrm{~m}$; In addition to making a phytosanitary and tortuosity analysis in the trees of an experiment of approximately 48 months, installed in Bonfinópolis-GO. The study was carried out between March 2018 and March 2019. In general terms, the $5 \times 5 \mathrm{~m}$ spacing showed the highest mean DAP, height and 
individual volume. Although there were no significant differences between the means of ICA for the diameter variable, it was possible to identify for the mean ICA of the variable height, that all the spacings presented results superior to that presented by the spacing $6 \times 6 \mathrm{~m}$. The $3 \times 3 \mathrm{~m}$ spacing showed higher volume per hectare, in view of its high population density.

KEYWORDS: Agroforestry, Commercial Plantation, Forestry Plantation.

\section{INTRODUÇÃO}

A combinação entre a ocorrência de áreas planas e uma tendência cada vez maior de substituição de pastagens degradadas por cultivos silviculturais, vem abrindo as portas para a silvicultura de espécies exóticas no estado de Goiás. Existem, porém, alguns fatores considerados limitantes, tais como a baixa fertilidade típica dos solos do cerrado, acidez elevada, déficit hídrico acentuado, condições de escassez de recursos do meio e competição intra e interespecífica advindos de espaçamentos erroneamente adensados (SCOLFORO, 1998; ALVES JR. et al., 2016).

O sucesso de um empreendimento florestal depende da escolha acurada de espécies florestais que apresentem grande potencial de adaptação e produção na região e da definição dos tratos silviculturais adequados, como por exemplo, o espaçamento. A escolha dos tratos silviculturais deve ser feita a fim de se otimizar a utilização de todos os fatores de produção disponíveis e ou limitantes no sítio (VILLA et al., 2016).

O espaçamento para ser classificado como ótimo deve ser capaz de potencializar as características da espécie. Maximizando a produção em volume, e mantendo padrões excelentes de tamanho, forma e qualidade. Realçando que a escolha do espaçamento adequado tem por objetivo proporcionar para cada indivíduo o espaço suficiente para se obter o crescimento máximo com a melhor qualidade e menor custo, sem deixar ao acaso fatores como a proteção do solo. (SCOLFORO, 1998).

As espécies do gênero Khaya sp. são pertencentes à família Meliaceae e são originárias de vários países da costa oeste do continente africano, como Benim, Camarões, Costa do Marfim, Gana, Nigéria e Togo (KLEIN et al., 2016). Em condições naturais o mogno africano ocorre desde 0 a $450 \mathrm{~m}$ de altitude, normalmente em locais úmidos. Esses países apresentam condições edafoclimáticas similares a algumas regiões brasileiras, podendo explicar a boa adaptação dessas espécies no Brasil (BRITO ; LEITE, 2018).

De acordo com a Associação Brasileira de Plantadores de Mogno Africano, estima-se a existência de mais de 10 mil hectares de Khaya sp. plantados atualmente no Brasil, tendo na maioria entre um a sete anos (ABPMA,2019). A madeira do Mogno africano é comercializada visando as indústrias de movelaria, construção naval e construção civil na parte decorativa. O interesse pela espécie vai além da madeira, visto que as sementes tem alto valor no mercado comercial (FERREIRA et al., 2015).

Somando as restrições impostas à comercialização do mogno brasileiro (Swietenia macrophylla King), à semelhança entre as propriedades da madeira das diferentes espécies de mogno e, à resistência da Khaya sp. ao ataque do microlepidóptero Hypsipyla grandella, que é a principal praga do mogno brasileiro, os plantios de mogno africano se expandiram em diferentes regiões do país (OFORI et al., 2007). 
No Brasil, até o presente momento há uma carência de informações sobre as tendências de crescimento de mogno africano. Segundo Ribeiro et al., (2017), os espaçamentos no Brasil tendem a serem mais amplos (4x6m, 5x5m, 6x6m, 5x8m), quando comparados aos plantios de K. ivorensis A.Chev. em partes da África que seguem o padrão de serem plantados em espaçamentos iniciais de $3 \times 3 \mathrm{~m}$.

Os objetivos desse trabalho foram quantificar o desenvolvimento dendrométrico e avaliar características qualitativas de plantas de mogno africano. Além disso, almeja-se indicar espaçamentos adequados a fim de fornecer melhor embasamento para os silvicultores que decidam por investir na espécie.

\section{Área Experimental}

\section{MATERIAL E MÉTODOS}

O experimento foi instalado em uma área experimental em BonfinópolisGO (16 35'49" de latitude Sul e 4916'39" de longitude Oeste e altitude de 780 m), cuja temperatura média anual é de $24,0{ }^{\circ} \mathrm{C}$. A média da umidade relativa do ar é de $71 \%$, e a precipitação média anual é de $1400 \mathrm{~mm}$ (INMET, 2019).

A região possui estações secas e chuvosas bem definidas (período chuvoso de outubro a abril, e período seco de maio a setembro). O clima da região é Aw, tropical de savana, megatérmico, segundo a classificação de Köppen (CASAROLI et al. 2019). A perda de água por evaporação, média anual, medida pelo tanque classe A, é da ordem de $1915 \mathrm{~mm}$.

O experimento foi instalado em 2015 , em cinco diferentes espaçamentos (5x5m;6x7m; 3x3m; 4x4m;6x6m) para avaliar o crescimento de 390 árvores de mogno africano, distribuídas em seis linhas por espaçamento, com aproximadamente 13 árvores por linha. As plantas foram produzidas a partir de sementes, no viveiro Mudas Nobres, localizado na BR060, Km 6, Goiânia-GO.

\section{Avaliação Dendrométrica}

A avaliação dos parâmetros dendrométricos consistiu em três fases, a primeira foi a medição do diâmetro a altura padrão (DAP), seguido pela determinação da variável altura pelo uso do clinômetro digital. A última fase consistiu no uso de um modelo ajustado para um experimento na região, para a determinação do volume para cada árvore. Após cada avaliação os dados foram computados no Microsoft Excel$/ \Theta$, o que permitiu ao final do período de avaliação do experimento calcular 0 Incremento Corrente Anual (ICA).

Para a avaliação do experimento foram selecionadas 78 árvores por espaçamento, totalizando 390 árvores, que foram por sua vez foram tratadas como parcelas de árvore única. Foi realizada a medição do diâmetro a altura padrão (DAP) com o auxílio de uma suta, e a altura foi tomada com o uso do clinômetro digital.

Com os dados da última avaliação foram realizados testes de variâncias para determinar se houve variação entre os espaçamentos. Como as parcelas estavam instaladas adjacentes umas às outras, foi desconsiderada quaisquer variações dentro dos espaçamentos, uma vez que essas variações se originariam de fatores genéticos, o que não foi o foco deste estudo.

Foram realizadas três medições no período de 12 meses. As medições foram realizadas com distância temporal de seis meses, sendo que a primeira medição foi realizada no mês de março de 2018 , que coincide com o final do período chuvoso. A segunda medição foi realizada no mês de setembro de 2018, que coincide com o final do período seco, e a terceira medição no mês de março de 2019. Os dados foram submetidos à análise de variância e posteriormente as 
médias foram comparadas pelo teste de Tukey a $5 \%$ de probabilidade. Para a realização das análises foi utilizado o Microsoft Excel® e o Software R.

\section{Avaliação de parâmetros qualitativos}

Foi realizada a avaliação visual de parâmetros de qualidade do fuste das árvores do experimento. A cada árvore foi atribuída uma nota de 0 a $2 \mathrm{com}$ respeito a sua tortuosidade, e uma nota de 0 a 2 com respeito aos aspectos fitossanitários.

A tortuosidade foi qualificada por meio de avaliação visual, sendo atribuída a nota 0 para árvores com o fuste retilíneo, 1 para árvores com um (1) desvio do tronco e 2 para árvores com dois ou mais desvios do tronco, observados através de um eixo hipotético traçado a 15 metros e 0 ㅇ da árvore de interesse.

A fitossanidade foi avaliada através de análise visual de presença de pragas, perda da casca e desrama prematura. Foi atribuída nota 0 para as árvores que não apresentaram nenhum ou muito pouco dos sinais indicados, nota 1 para as árvores que apresentaram sinais de infestação, comprometendo o desenvolvimento e logicamente a produtividade final.

\section{RESULTADOS E DISCUSSÃO}

As tabelas abaixo, apresentam as médias por espaçamento das três medições realizadas no período de 12 meses, sendo que a Tabela 1 apresenta os dados de incremento e as médias de diâmetro a altura padrão (DAP). Para tal variável, foi identificada superioridade das médias atuais nos espaçamentos $5 \times 5 \mathrm{~m}$ e $6 \times 7 m$. No I.C.A para a variável DAP não houve diferença significativa entre os espaçamentos.

TABELA 1. Valores médios de DAP $(\mathrm{cm})$, I.C.A $(\mathrm{cm})$ e teste Tukey $5 \%$, para as medições realizadas entre março de 2018 e março de 2019.

\begin{tabular}{ccccc}
\hline Tratamentos & DAP $(\mathrm{mar} / 18)$ & DAP $(\mathrm{set} / 18)$ & DAP $(\mathrm{mar} / 19)$ & ICA.DAP \\
\hline $5 \times 5$ & $12,48 \mathrm{a}$ & $13,44 \mathrm{a}$ & $15,03 \mathrm{a}$ & $2,55 \mathrm{a}$ \\
$6 \times 7$ & $11,84 \mathrm{a}$ & $12,76 \mathrm{a}$ & $14,30 \mathrm{a}$ & $2,46 \mathrm{a}$ \\
$3 \times 3$ & $10,02 \mathrm{~b}$ & $10,95 \mathrm{~b}$ & $12,36 \mathrm{~b}$ & $2,34 \mathrm{a}$ \\
$4 \times 4$ & $10,42 \mathrm{~b}$ & $11,33 \mathrm{~b}$ & $12,90 \mathrm{~b}$ & $2,54 \mathrm{a}$ \\
$6 \times 6$ & $10,70 \mathrm{~b}$ & $11,58 \mathrm{~b}$ & $13,18 \mathrm{~b}$ & $2,48 \mathrm{a}$ \\
\hline
\end{tabular}

Em que: CV\%: 16,69; 16,48; 15,08 e; 26,38, para DAP (mar/18), DAP (set/18), DAP (mar/19) e ICA. DAP, respectivamente. Diferença mínima significativa a $95 \%$ de probabilidade de: 0,$81 ; 0,86 ; 0,89$ e 0,29, para DAP (mar/18), DAP (set/18), DAP (mar/19) e ICA. DAP, respectivamente.

Os dados avaliados mostram o padrão de crescimento e desenvolvimento populacional referente a cada espaçamento. Em plantios mais adensados é de se esperar que as árvores priorizem o desenvolvimento vertical, em virtude da competição intraespecífica pela luz (VIDAURRE et al.,2015). Nessas condições a árvore tenta ocupar o dossel, assim ampliando ao máximo a superfície foliar e suprindo a necessidade de produtos oriundos da fotossíntese, estimulando, portanto, o crescimento em altura.

Já para a variável altura, o espaçamento 5x5m apresentou uma média atual superior aos demais, como apresentado na Tabela 2. Além disso, o incremento corrente anual para a variável altura apenas apresentou diferenças significativas entre os espaçamentos $3 \times 3 \mathrm{~m}$ e $6 \times 6 \mathrm{~m}$, sendo que o espaçamento mais adensado apresentou maior média que o menos adensado. 
TABELA 2. Valores médios de Altura $(\mathrm{m})$, I.C.A $(\mathrm{m})$ e teste Tukey $5 \%$, para as medições realizadas entre março de 2018 e março de 2019.

\begin{tabular}{lcccc}
\hline Tratamentos: & $\mathrm{H}(\mathrm{mar} / 18)$ & $\mathrm{H}(\mathrm{set} / 18)$ & $\mathrm{H}(\mathrm{mar} / 19)$ & $\mathrm{ICA} . \mathrm{H}$ \\
\hline $5 \times 5$ & $9,33 \mathrm{a}$ & $9,94 \mathrm{a}$ & $11,65 \mathrm{a}$ & $2,33 \mathrm{ab}$ \\
$6 \times 7$ & $8,22 \mathrm{~b}$ & $8,78 \mathrm{~b}$ & $10,36 \mathrm{~b}$ & $2,15 \mathrm{ab}$ \\
$3 \times 3$ & $7,43 \mathrm{c}$ & $7,58 \mathrm{c}$ & $10,04 \mathrm{~b}$ & $2,61 \mathrm{a}$ \\
$4 \times 4$ & $7,42 \mathrm{c}$ & $7,54 \mathrm{c}$ & $9,82 \mathrm{~b}$ & $2,44 \mathrm{ab}$ \\
$6 \times 6$ & $7,9 \mathrm{bc}$ & $8,45 \mathrm{~b}$ & $10,02 \mathrm{~b}$ & $2,12 \mathrm{~b}$ \\
\hline
\end{tabular}

Em que: CV\%: 19,78; 19,49; 18,13 e; 47,36, para H (mar/18), H (set/18), H (mar/19) e ICA.H, respectivamente. Diferença mínima significativa a $95 \%$ de probabilidade de: 0,$70 ; 0,72 ; 0,82$ e 0,49 , para $\mathrm{H}$ (mar/18), $\mathrm{H}$ (set/18), H (mar/19) e ICA. H, respectivamente.

$\mathrm{Na}$ análise dos dados referentes ao diâmetro médio da árvore, os resultados práticos apontam a superioridade das árvores dos espaçamentos $5 \times 5 \mathrm{~m}$ e $6 \times 7 \mathrm{~m}$, que não diferiram entre si, sobre as demais. Os resultados obtidos na análise dos dados de altura média, foram contraditórios aos esperados teoricamente, agrupando os espaçamentos mais adensados, $3 \times 3 \mathrm{~m}$ e $4 \times 4 \mathrm{~m}$, em uma classe inferior a um espaçamento mais amplo, $5 \times 5 \mathrm{~m}$.

A primeira explicação para tal resultado, se baseia no fato do plantio ser relativamente jovem, não havendo tido tempo suficiente para expressar respostas fisiológicas às condições impostas pelo espaçamento. Um fato que sustenta tal argumento é o começo de uma diferenciação das médias de ICA entre os espaçamentos mais adensados e menos adensados, representados por sua vez pelo espaçamento $3 \times 3 \mathrm{~m}$ e $6 \times 6 \mathrm{~m}$, respectivamente.

Uma segunda explicação, é baseada no fato das espécies de Khaya sp. serem de crescimento médio, exigentes de luz (ALVES JR. et al., 2016). Essa exigência de luz, teria afetado os espaçamentos mais adensados de forma negativa, diminuindo a capacidade de incremento em altura na fase do arranque inicial, o que vem se refletindo ao longo do desenvolvimento do plantio, podendo, porém, ser estabilizado conforme o amadurecimento do mesmo.

A terceira explicação se baseia em características do solo e se baseia em apontar um esgotamento dos recursos nutricionais disponíveis, ocasionados pela alta densidade populacional nos espaçamentos de $3 \times 3 \mathrm{~m}$ e $4 \times 4 \mathrm{~m}$, e ainda baixa taxa de ciclagem de nutrientes, como responsável direto pelo baixo crescimento vertical do plantio. Com as informações de DAP e Altura, foi possível realizar o cálculo do volume por árvore e por hectare. O cálculo do volume é em suma a representatividade da produtividade do tratamento escolhido.

Para o cálculo do volume com casca, foi utilizado um modelo volumétrico ajustado, obtido em um estudo prévio em uma área experimental na região de Paranoá-DF a aproximadamente $240 \mathrm{~km}$ de distância de Bonfinópolis. A seguir, a Tabela 3 apresenta os dados médios de volume unitário e volume extrapolado para 1 hectare para cada tratamento e o Teste Tukey para tais médias. 
TABELA 3. Teste Tukey (5\%) para volume unitário $\left(\mathrm{m}^{3}\right)$ e para volume por hectare $\left(\mathrm{m}^{3} / \mathrm{ha}\right)$ por espaçamento.

\begin{tabular}{lcc}
\hline Tratamentos: & Volume Individual $\left(\mathrm{m}^{3}\right)$ & Volume/Hectare $\left(\mathrm{m}^{3} / \mathrm{ha}\right)$ \\
\hline $5 \times 5$ & $0,11 \mathrm{a}$ & $42,45 \mathrm{~b}$ \\
$6 \times 7$ & $0,09 \mathrm{~b}$ & $20,25 \mathrm{c}$ \\
$3 \times 3$ & $0,07 \mathrm{c}$ & $76,92 \mathrm{a}$ \\
$4 \times 4$ & $0,07 \mathrm{c}$ & $41,30 \mathrm{~b}$ \\
$6 \times 6$ & $0,08 \mathrm{bc}$ & $21,36 \mathrm{c}$ \\
\hline
\end{tabular}

Em que: CV\%: 37,88 e 50,32, para Volume em $\mathrm{m}^{3}$ e $\mathrm{m}^{3} / \mathrm{ha}$, respectivamente. Diferença mínima significativa à $95 \%$ de probabilidade de: 0,01 e 8,89 , para Volume em $\mathrm{m}^{3}$ e $\mathrm{m}^{3} / \mathrm{ha}$, respectivamente.

Quando calculados os valores de volume individual houve a predominância do espaçamento $5 \times 5 \mathrm{~m}$ que alcançou a média de $0,11 \mathrm{~m}^{3}$ por árvore, seguido pelo espaçamento $6 \times 7 \mathrm{~m}$ e $6 \times 6 \mathrm{~m}$, sendo que o último desses não se diferenciou dos demais. Já na extrapolação para volume por hectare, em que é levado em consideração a quantidade máxima de árvores passíveis de implantação, foi observada a superioridade do espaçamento $3 \times 3 \mathrm{~m}$ que alcançou a média de $76,92 \mathrm{~m}^{3} / \mathrm{ha}$, seguido pelos espaçamentos $5 \times 5 \mathrm{~m}$ e $4 \times 4 \mathrm{~m}$ que se diferenciaram dos restantes. Porém, o maior volume em plantios mais densos é esperado devido ao maior número de plantas (SILVA et al., 2016).

Ao final do ciclo, é de se esperar que a produtividade total de um espaçamento menos adensado se equipare à produtividade de um espaçamento mais adensado. $O$ fator que diferia os povoamentos seriam as dimensões e a densidade da madeira final, e a escolha sobre o tipo de regime ao qual fazer a implantação do plantio ficaria a cargo do uso da madeira resultante do plantio (SCOLFORO, 1998).

Em um plantio de Pinus taeda L., Lima et al. (2013) apontaram que a escolha do espaçamento está vinculada com o objetivo final do plantio. Se o objetivo do plantio for apenas o volume bruto, espaçamentos mais adensados são mais indicados. Porém, se o objetivo estiver vinculado com as dimensões individuais de cada tora, deve-se optar por espaçamentos mais amplos.

Durante a última medição foi feita uma avaliação visual de caracteres qualitativos na área experimental, quanto a tortuosidade do fuste e a presença ou não de pragas e ou sintomas de doenças das árvores. Os resultados obtidos estão apresentados em forma percentual na Tabela 4.

TABELA 4. Percentuais de árvores tortuosas e árvores afetadas com problemas fitossanitários por espaçamento

\begin{tabular}{lccc|cc}
\hline \multirow{2}{*}{ Espaçamentos } & \multicolumn{3}{c|}{ Tortuosidade } & \multicolumn{2}{c}{ Fitossanidade } \\
\cline { 2 - 6 } & 0 & 1 & 2 & 0 & 1 \\
\hline $5 \times 5$ & 51,3 & 34,6 & 14,1 & 92,3 & 7,7 \\
$6 \times 7$ & 51,3 & 33,3 & 15,4 & 89,7 & 10,3 \\
$3 \times 3$ & 50,0 & 33,8 & 16,2 & 88,5 & 12,2 \\
$4 \times 4$ & 55,7 & 31,4 & 12,9 & 87,2 & 14,3 \\
$6 \times 6$ & 55,8 & 36,4 & 7,8 & 93,6 & 5,2 \\
\hline
\end{tabular}


Quanto aos dados qualitativos, não houveram diferenças quanto aos parâmetros analisados. Mesmo sendo árvores de origem seminal, o fuste até o momento se apresenta retilíneo e sem reentrâncias em pelo menos $50 \%$ dos casos. Os parâmetros fitossanitários do povoamento apresentam a concentração dos indivíduos na classe de árvores saudáveis. As árvores que foram classificadas com rank 1 estavam mortas em pé ou eram árvores mortas que já não estavam no espaçamento. Sendo assim, não foi observada a presença de nenhuma praga ou infestação que venha a ser motivo de preocupação para o responsável técnico do plantio.

\section{CONCLUSÃO}

- O espaçamento 5x5m apresentou melhores médias de DAP, Altura e Volume Individual.

- O espaçamento 3x3m apresentou melhor média para Volume em um hectare.

- Não houveram diferenças significativas entre os espaçamentos, quanto à tortuosidade e mortalidade.

\section{REFERÊNCIAS}

ABPMA - Associação Brasileira de Produtores de Mogno Africano. Khaya ivorensis. 2019. Disponível em: <http://abpma.org.br>. Acesso em: 26 maio 2019.

ALVES JÚNIOR, J.; BARBOSA, L.H.A.; CASAROLI, D.; EVANGELISTA, A.W.P.; COSTA, F. Crescimento de mogno africano submetido a diferentes níveis de irrigação por microaspersão. Irriga, v. 21, n. 3, p. 466, 2016. Disponível em: $<$ http://200.145.140.50/index.php/irriga/issue/view/85>.

DOI: <https://doi.org/10.15809/irriga.2016v21n3p466-480>.

BRITO, D. S.; LEITE, E. F. Análise do uso e ocupação da terra aplicada ao diagnóstico físico-conservacionista-DFC-da Bacia Hidrográfica do Rio Água Suja, Tocantins-Brasil. Revista Sítio Novo, v. 2, n. 1, p. 5-22, 2018. Disponível em <http://sitionovo.ifto.edu.br/index.php/sitionovo/article/view/68>.

CASAROLI, D., SÉRVULO, A. C.; VELLAME, L. M.; ALVES JÚNIOR, J. EVANGELISTA, A. W. P., et al. Transpiration and growth of young African mahogany plants subject to different water regimes. International Journal Biometeorology, 2019. Disponível em: <https://link.springer.com/content/pdf/10.1007\%2Fs00484-01901771-5.pdf>. DOI: <https://doi.org/10.1007/s00484-019-01771-5>.

FERREIRA, D. C.; POMPEU, G.S.S.; FONSECA, J.R.; DOS SANTOS, J.C. Sistemas agroflorestais comerciais em áreas de agricultores familiares no município de Altamira, Pará. Revista Brasileira de Agroecologia, v. 9, n. 3, 2015. ISSN 19809735. Disponível em: <http://revistas.abaagroecologia.org.br/index.php/rbagroecologia/article/view/15493>. Acesso em: 04 oct. 2019.

INMET - Instituto Nacional de Meteorologia. Normais climatológicas do Brasil Período 1981-2010 <http://www.inmet.gov.br/portal/index.php?r=clima/normaisClimatologicas $>$. Acesso em: 27 Sept,2019. 
KLEIN, D. R.; ANDRADE, M. M.; DERENGOSKI, J. A.; DUARTE, E.; KREFTA, S. $M$, et al. Aspectos gerais e silviculturais de Cordia americana, Aspidosperma polyneuron, Toona ciliata e Khaya spp. Revista de Ciências Agroveterinárias, v. 15, n. 2, p. 155-164, 2016. Disponível em: $<$ http://www.revistas.udesc.br/index.php/agroveterinaria/article/view/2238117115220 16155>. DOI: <http://dx.doi.org/10.5965/223811711522016155>.

LIMA, R.; INOUE, M.T.; FIGUEREDO FILHO, A.; DE ARAUJO, A.J.; MACHADO, S.M. Efeito do espaçamento no desenvolvimento volumétrico de Pinus taeda L. Floresta Ambiente. v. 20, n. 2, p. 223-230, June 2013. Disponível em: $<$ http://www.scielo.br/scielo.php?script=sci_arttext\&pid=S2179-

80872013000200010\&Ing=en\&nrm=iso>. Access on 27 Sept. 2019. DOI: <http://dx.doi.org/10.4322/floram.2013.001>.

OFORI, D. A.; OPUNI-FRIMPONG, E.; COBBINAH, J. R. Provenance variation in Khaya species for growth and resistance to shoot borer Hypsipyla robusta. Forest Ecology and Management, v. 242, p. 438-443, 2007. Disponível em: <https://reader.elsevier.com/reader/sd/pii/S0378112707000850?token=4CDFF4B204 8544A0BB99E97D383809D0B82698ADB6C7D5993104522E785FBB64030D311046 BF9FAE7A7E105AB89ADD08 >. DOI: <10.1016/j.foreco.2007.01.090>.

RIBEIRO, A.; FERRAZ FILHO, A. C.; SCOLFORO, J. R. S., O cultivo do mogno africano (Khaya spp.) e o crescimento da atividade no Brasil. Floresta e Ambiente, v. 24, Seropédica, 2017 Epub 27-Abr-2017. Disponível em: $<$ http://www.scielo.br/scielo.php?script=sci_arttext\&pid=S2179-

80872017000100201\&lng=pt\&tIng=pt>. DOI: <http://dx.doi.org/10.1590/21798087.076814>.

SILVA, R. S.; VENDRUSCOLO, D. G. S.; ROCHA, J. R. M.; CHAVES, A. G. S.; SOUZA, H. S., et al. Desempenho silvicultural de Tectona grandis L. f. em diferentes espaçamentos em Cáceres, MT. Floresta e Ambiente, v. 23, n. 3, p. 397-405, 2016. Disponível em <http://www.scielo.br/pdf/floram/2016nahead/2179-8087-floram-21798087143015.pdf>. DOI: <http://dx.doi.org/10.1590/2179-8087.143015>.

SCOLFORO, J. R. S., Manejo Florestal. Lavras: Editora UFLA, 1998. 438p.

VIDAURRE, G. B.; NUTTO, L.; FRANÇA, F. J. N.; BRAZ, R. L.; WATZLAWIC L. F. et al. Tensão de crescimento no lenhoff de Eucalyptus benthamii e sua relação com características dendrométricas em diferentes espaçamentos. Floresta e Ambiente, v. 22, n. 3, p. 408-415, 2015. Disponível em: <http://www.scielo.br/scielo.php?script=sci_arttext\&pid=S2179-

80872015000300408\&lng=pt\&tIng=pt>. _ DOI: <http://dx.doi.org/10.1590/21798087.044413>.

VILLA, E. B.; PEREIRA, M. G.; ALONSO, J. M.; BEUTLER, S. J.; LELES, P. S. S., Aporte de serapilheira e nutrientes em área de restauração florestal com diferentes espaçamentos de plantio. Floresta e Ambiente, v. 23, n. 1, p. 90-99, 2016. Disponível em: <http://www.scielo.br/pdf/floram/2016nahead/2179-8087-floram-21798087067513.pdf>. DOI: <http://dx.doi.org/10.1590/2179-8087.067513>. 